\title{
Topological magnetotorsional effect in Weyl semimetals
}

\author{
Long Liang $\oplus^{1,2}$ and Teemu Ojanen $\oplus^{2,3}$ \\ ${ }^{1}$ Department of Applied Physics, Aalto University School of Science, FI-00076 Aalto, Finland \\ ${ }^{2}$ Computational Physics Laboratory, Physics Unit, Faculty of Engineering and Natural Sciences, \\ Tampere University, P.O. Box 692, FI-33014 Tampere, Finland \\ ${ }^{3}$ Helsinki Institute of Physics, P.O. Box 64, FI-00014 Helsinki, Finland
}

(Received 17 December 2019; accepted 30 March 2020; published 23 April 2020)

\begin{abstract}
In this Rapid Communication we introduce a thermal magnetotorsional effect (TME) as a topological response in magnetic Weyl semimetals. We predict that magnetization gradients perpendicular to the Weyl node separation give rise to temperature gradients depending only on the local positions of the Weyl nodes. The TME is a consequence of magnetization-induced effective torsional spacetime geometry and the finite-temperature NiehYan anomaly. Similarly to the anomalous Hall effect and chiral anomaly, the TME has a universal materialindependent form. We predict that the TME can be observed in the magnetic Weyl semimetal $\mathrm{EuCd}_{2} \mathrm{As}_{2}$.
\end{abstract}

DOI: 10.1103/PhysRevResearch.2.022016

Introduction. The cross-fruition between condensed matter and high-energy physics has become a central theme in contemporary research. Topological Weyl and Dirac semimetals [1] provide a particularly rich example of this interplay. These systems offer a versatile playground to study the phenomenology of relativistic fermions in the presence of gauge fields and curved spacetimes as well as inspire novel electronic applications. Due to the flexibility of condensed matter systems, there are no fundamental limitations to realize predictions that have remained hypothetical in the high-energy context. A fascinating example of such predictions is the Nieh-Yan anomaly [2-8] of chiral fermions in torsional spacetimes which has recently been proposed as a source of universal effects in condensed matter systems.

Significant interest in topological semimetals is focused on their remarkable material-independent properties resulting from momentum-space Berry monopoles and field theory anomalies. In the present Rapid Communication, we report the discovery of an addition to the previously known topological responses in Weyl semimetals. We introduce the thermal magnetotorsional effect (TME) which gives rise to energy currents and temperature gradients as a response to magnetic gradients. This effect results from two important factors. First, as depicted in Fig. 1(a), the Weyl fermions couple to spacetime geometry through frame fields. As established in Refs. $[9,10]$, smoothly varying magnetization will give rise to a low-energy description in terms of locally varying frame fields. As discussed in the present work, the effective geometry encoded in frame fields admits nonvanishing torsion. Another key ingredient is the Nieh-Yan anomaly of chiral fermions in torsional spacetimes. Until recently, the physical

Published by the American Physical Society under the terms of the Creative Commons Attribution 4.0 International license. Further distribution of this work must maintain attribution to the author(s) and the published article's title, journal citation, and DOI. consequences of the Nieh-Yan anomaly [2,3] have remained controversial due to the explicit appearance of a nonuniversal high-energy cutoff in physical predictions. However, several recent studies [4-7] have confirmed its universal character by identifying temperature as the appropriate substitute for the high-energy cutoff.

We first present a derivation of an effective low-energy theory of magnetic Weyl materials with torsional geometry. We proceed by showing how the TME results from combining the effective geometry with the finite-temperature Nieh-Yan anomaly. Then we discuss the striking physical consequences of the TME by studying a system depicted in Fig. 1(b). By connecting a magnetic Weyl material to a thermal bath at one end, the relative temperature drop in the system is given by the relative shift of the node separation $T_{h} / T_{c}=\sqrt{k_{W}^{c} / k_{W}^{h}}$. This remarkable relation has the same universal form in all Weyl materials and is independent on the specific spatial profile of the magnetic texture. We discuss how this relation can be experimentally probed in the magnetic Weyl material $\mathrm{EuCd}_{2} \mathrm{As}_{2}$ [11-13].

Torsional spacetimes in magnetic Weyl semimetals. We first outline the general mechanism for the emergent torsional geometry in time-reversal (TR) breaking Weyl semimetals. We consider a four-band parent Hamiltonian that breaks the TR symmetry,

$$
H=k_{i} \gamma_{i}+m \gamma_{4}+\mathbf{M}(\mathbf{r}) \cdot \mathbf{b},
$$

where $\gamma_{i}$ with $i=1,2,3,4$ denotes the four Hermitian $\gamma$ matrices satisfying anticommutation relations $\left\{\gamma_{i}, \gamma_{j}\right\}=2 \delta_{i j}$ with $\delta_{i j}$ being the Kronecker delta function and $\mathbf{b}=\left(\gamma_{23}, \gamma_{31}, \gamma_{12}\right)$ with $\gamma_{i j}=-i\left[\gamma_{i}, \gamma_{j}\right] / 2$. Since the momentum is odd under the TR, $\gamma_{1,2,3}$ and $\mathbf{b}$ are also odd. The $\mathbf{M}(\mathbf{r}) \cdot \mathbf{b}$ term breaks the TR symmetry, and $\mathbf{M}(\mathbf{r})=$ $M(\mathbf{r})[\sin \theta(\mathbf{r}) \cos \phi(\mathbf{r}), \sin \theta(\mathbf{r}) \sin \phi(\mathbf{r}), \cos \theta(\mathbf{r})] \quad$ corresponds to three-dimensional (3D) magnetization or any field that transforms as magnetization under TR. It can be used to describe real materials such as $3 \mathrm{D}$ topological insulators 


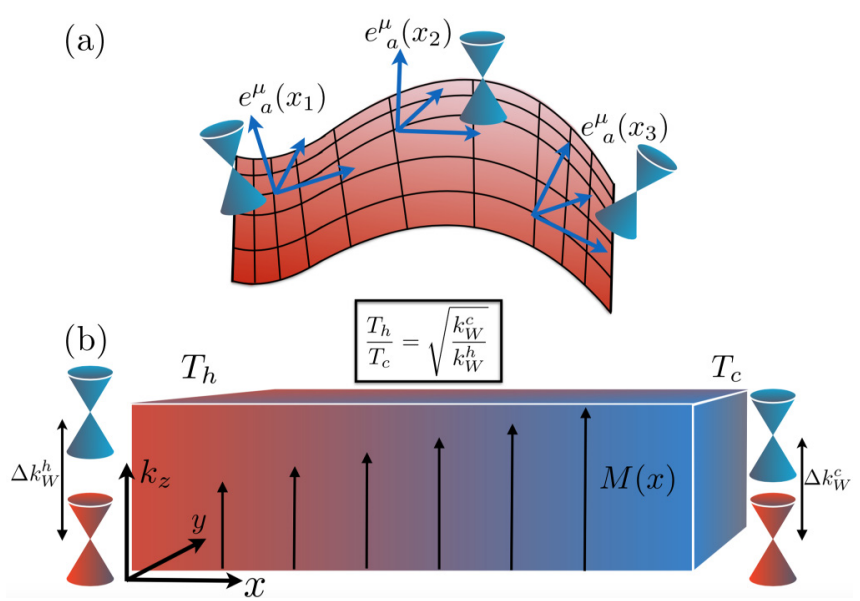

FIG. 1. (a) Weyl fermions couple to spacetime geometry through the frame fields $e^{\mu}{ }_{a}$. Inhomogeneous magnetization gives rise to locally varying frame fields in Weyl semimetals. (b) The thermal magnetotorsional effect gives rise to a temperature gradient as a response to a magnetic gradient perpendicular to the Weyl node separation $\Delta k_{W}$. Placing one end of a sample in contact with a heat bath, the relative temperature drop between the ends is given by the relative shift of the node separation.

(TIs) with a magnetic texture [14], topological insulator heterostructures $[15,16]$, or ferromagnetic Weyl semimetals discovered recently [11-13,17-20].

Following the general method introduced in Refs. $[9,10]$, we block diagonalize the parent Hamiltonian [for details, see the Supplemental Material (SM) [21]]. This leads to an effective Weyl Hamiltonian,

$$
H_{W}=d_{a}(\mathbf{k}, \mathbf{r}) \sigma^{a},
$$

where $a=0,1,2,3, \sigma^{0}$ is the $2 \times 2$ unit matrix, $\sigma^{1,2,3}$ are the Pauli matrices, $d_{1}=\cos \theta \cos \phi k_{x}+\cos \theta \sin \phi k_{y}-$ $\sin \theta k_{z}, \quad d_{2}=-\sin \phi k_{x}+\cos \phi k_{y}, \quad d_{3}=M-m-\left(\kappa_{3}^{2}+\right.$ $\left.f^{2}\right) / 2 m, \quad d_{0}=-f \kappa_{3} / m \quad$ with $\quad \kappa_{3}=\cos \phi \sin \theta k_{x}+$ $\sin \phi \sin \theta k_{y}+\cos \theta k_{z}, \quad$ and $\quad f=\left(\partial_{z} \phi+\cos \phi \partial_{y} \theta-\right.$ $\left.\sin \phi \partial_{x} \theta\right) / 2$. The Weyl points are located at $\pm \mathbf{K}_{W}= \pm K_{W}(\sin \theta \cos \phi, \sin \theta \sin \phi, \cos \theta) \quad$ with $\quad K_{W}=$ $\sqrt{2 m(M-m)-f^{2}}$. We assume $2 m(M-m)>f^{2}$ such that there are always two well-separated Weyl points.

Expanding Eq. (2) around the Weyl point $\mathbf{K}_{W}$, we obtain the Hamiltonian for the left-handed Weyl fermion,

$$
H_{L} \approx \frac{1}{2}\left\{v_{a}^{i}, k_{i}-K_{W, i}\right\} \sigma^{a}-K_{W, 0} \sigma^{0},
$$

where $K_{W, 0}=f K_{W} / m$ is the energy shift of the Weyl point and $v_{a}^{i}(\mathbf{r})=\left.\frac{\partial d_{a}}{\partial k_{i}}\right|_{\mathbf{K}_{W}}$ is the position-dependent Fermi velocity. It is convenient to introduce $v^{0}{ }_{0}=-1$ and $v_{1,2,3}^{0}=0$ such that $v$ can be written as a $4 \times 4$ matrix. The explicit expressions for $v^{\mu}{ }_{a}$ are given in SM. The effective Weyl Hamiltonian is similar to the one induced by elastic deformations [22], where the position-dependent Fermi velocities are related to the strain instead of magnetization. However, an important difference is that the spacetime mixed components $v_{0}^{i}$ are absent in the strained Weyl systems, whereas in inhomogeneous magnetic Weyl semimetals the mixed term can exist and give rise to exotic physics [10].
The action corresponding to the left-handed Weyl fermion takes the form (see SM)

$$
S_{L}=\int d^{4} x e \psi_{L}^{\dagger} e^{\mu}{ }_{a}\left(i \partial_{\mu}+A_{5, \mu}+i \frac{T_{\mu}}{2}\right) \sigma^{a} \psi_{L},
$$

where $\psi_{L}$ is a two-component spinor, $e \equiv \operatorname{det}\left(e_{\mu}{ }^{a}\right)=$ $\left(\operatorname{det} v_{a}^{\mu}\right)^{1 / 3}$ gives the invariant volume element, and $e^{\mu}{ }_{a}=$ $e^{-1} v^{\mu}{ }_{a}$ is the frame field with the coordinate index denoted by $\mu=t, x, y, z$ and the Lorentz index by $a=0,1,2,3$. The emergent metric is given $g_{\mu \nu}=e_{\mu}{ }^{a} e_{\nu}{ }^{b} \eta_{a b}$ with $\eta_{a b}=$ $\operatorname{diag}(-1,1,1,1)$ and $e_{\mu}{ }^{a}$ being the coframe field satisfying $e_{\mu}{ }^{a} e^{v}{ }_{a}=\delta_{\mu}^{v}$ and $e_{\mu}{ }^{a} e^{\mu}{ }_{b}=\delta_{b}^{a}$. The position of the Weyl point is given by the "axial gauge field" $A_{5, \mu}=K_{W, \mu}$. Comparing to the standard action of a spinor field in gravitational fields [23], the spin connection is absent in Eq. (4). As a result, the curvature also vanishes and the torsion two form $T^{a}$ is simply given by the exterior derivative of the coframe one form $e^{a}$, $T^{a}=d e^{a}$. The torsion is in general nonzero and therefore the inhomogeneous magnetization induces a low-energy Weyl fermion living in a Weitzenböck spacetime, which is used in teleparallel gravity theory [24]. The coframe field $e^{a}$ can also be viewed as a gauge potential, and then the torsion is the field strength corresponding to the potential. Note that it is the trace of the torsion tensor $T_{\mu}=T_{\mu \nu}{ }^{\nu}=e^{-1} e_{\mu}{ }^{a} \partial_{\nu}\left(e e^{v}{ }_{a}\right)$ that appears in the action. We consider a static magnetization so the frame fields are time independent, but in general it is possible to realize time-dependent frame fields. Thus, in the following derivation of the TME we will allow for time-dependent frame fields and formulate the general theory in a covariant form.

Finite-temperature mixed chiral-torsional anomaly. The low-energy theory Eq. (4) has far-reaching physical consequences. Here, we focus on the torsion-induced effects which have attracted significant attention recently [3-6,8,25-35]. It has been shown that the torsion gives a contribution to the chiral anomaly [2] through the Nieh-Yan term [36], which in the absence of spin connection reads

$$
N=\frac{\varepsilon^{\mu \nu \rho \sigma}}{4} T_{\mu \nu}{ }^{a} T_{\rho \sigma a}=2 \mathbf{E}^{a} \cdot \mathbf{B}_{a},
$$

where $\varepsilon^{\mu \nu \rho \sigma}$ is the totally antisymmetric symbol with the convention $\varepsilon^{t x y z}=1$, and $\mathbf{E}^{a}$ and $\mathbf{B}^{a}$ are the torsional electric and magnetic fields, respectively. The Nieh-Yan term looks similar to the celebrated Adler-Bell-Jackiw anomaly [37,38]. However, a fundamental difference is that the Nieh-Yan term has the dimension of inverse length squared $\left[L^{-2}\right]$ while the electromagnetic $\mathbf{E} \cdot \mathbf{B}$ term has the dimension of $\left[L^{-4}\right]$. Consequently, the coefficient of the Nieh-Yan contribution to the chiral anomaly has the dimension of $\left[L^{-2}\right]$. It was pointed out that the coefficient depends on the high-energy cutoff in Weyl semimetals [3] and therefore makes the contribution superficially nonuniversal. Recently, it was suggested [4,5] that the temperature can also play the role of cutoff since it has the correct dimension, and the temperature-dependent contribution of the Nieh-Yan anomaly takes a universal form,

$$
\partial_{\mu}\left(e J_{5}^{\mu}\right)=\frac{T^{2}}{12} N
$$


This result has been verified by a direct calculation of the chiral charge density induced by torsional Landau levels, establishing the Nieh-Yan anomaly as a universal Fermi-surface effect at finite temperatures. Furthermore, it has been argued that in addition to temperature, the coefficient contains a dimensionless central charge of (1+1)D Dirac fermions [6]. Since the central charge is also related to the $(1+1) \mathrm{D}$ gravitational anomaly [39], there could be a connection between the thermal Nieh-Yan anomaly in $(3+1) \mathrm{D}$ and the gravitational anomaly in $(1+1) \mathrm{D}$ which is yet to be revealed, moreover, temperature-dependent torsional anomalies may also appear in other dimensions [8]. However, the most important insight from the recent activity is the emergence of a simple physical picture of the Nieh-Yan contribution to the chiral anomaly in terms of torsional Landau levels and its universal nature at finite temperature.

From the thermal Nieh-Yan anomaly, we find a contribution to the effective action,

$$
S_{\text {anomaly }}=\frac{T^{2}}{12} \int d^{4} x \varepsilon^{\mu \nu \rho \sigma} A_{5 \mu} e_{\nu}{ }^{a} \partial_{\rho} e_{\sigma a} .
$$

This effective action leads to a chiral current $e j_{5}^{\mu}=$ $T^{2} \varepsilon^{\mu \nu \rho \sigma} e_{\nu}{ }^{a} \partial_{\rho} e_{\sigma a} / 12$, which is consistent with the current obtained from linear response calculations [31,32] and therefore provides additional confirmation on the validity of the anomaly action. The original action, Eq. (3), exhibits the Nieh-Yan-Weyl rescaling symmetry [27] $e^{a} \rightarrow \exp (\Lambda) e^{a}$ which does not carry over to the quantum theory because of the anomaly. The overall normalization of the frame fields in the effective action is fixed by requiring that the chiral densities resulting from the direct torsional Landau level calculation are equal to the one obtained from the effective action. Thus we find that the frame fields should be normalized so that the volume element is unity $e=1$ (see the SM for details). The zero-temperature counterpart of Eq. (7) was discussed in Refs. [3,25,27,28] in the context of lattice dislocations. However, due to the cutoff-dependent and impractical nature of the physical consequences, the experimental signatures of the Nieh-Yan anomaly have remained unclear. In contrast, as shown below, the finite-temperature anomaly gives rise to profound universal effects that can be observed experimentally.

Topological magnetotorsional effect. Taking variation of the effective action Eq. (7) with respect to the coframe fields, we obtain the anomalous energy-momentum current,

$$
e T^{\mu}{ }_{a}=-\frac{T^{2}}{6} \varepsilon^{\mu \nu \rho \sigma} A_{5 v} \partial_{\rho} e_{\sigma a}+\frac{T^{2}}{12} \varepsilon^{\mu \nu \rho \sigma} \partial_{\nu} A_{5 \rho} e_{\sigma a} .
$$

The second term on the right-hand side of the above equation is omitted in Ref. [6], but in our case it is of the same order as the first term since the frame fields and axial gauge potential have the same physical origin. It can also be understood as the 0th chiral pseudo-Landau-level contribution to the energy current [40]. For $a=0$, we get the energy current. The physical energy-momentum current corresponds to $e T_{v}^{\mu}$, which is obtained by converting the lower Lorentz index to the spacetime index with the help of coframe fields [41], but this does not affect our main result, so we use $e T^{\mu}{ }_{a}$ for simplicity.
Assuming $A_{5, t}=0$, then the energy current reads $\left(J_{\varepsilon}^{i} \equiv e T^{i}{ }_{0}\right)$

$$
\mathbf{J}_{\varepsilon}=-\frac{T^{2}}{6} \mathbf{A}_{5} \times \mathbf{E}_{0}-\frac{T^{2}}{12} \mathbf{B}_{5} e_{t 0},
$$

where $\mathbf{B}_{5}=\boldsymbol{\nabla} \times \mathbf{A}_{5}$ is the chiral magnetic field and $E_{i, 0}=$ $\partial_{i} e_{t 0}-\partial_{t} e_{i 0}$ is the torsional electric field. Remarkably, since the chiral vector potential $\mathbf{A}_{5}=\left(k_{W} / M\right) \mathbf{M}(\mathbf{r})$ and the frame fields $e_{\mu 0}$ are directly given in terms of the local magnetization $\mathbf{M}(\mathbf{r})$, Eq. (9) in fact expresses the energy current as a response to magnetization. This gives rise to a different magnetothermal response in Weyl semimetals. We call relation (9), combined with the expressions for magnetization-induced $\mathbf{A}_{5}$ and $e_{\mu 0}$, as the TME. The energy current is perpendicular to the separation between the Weyl points.

On the other hand, the temperature gradient is also a driving force of the energy current. Temperature gradients can be formally identified with an extra torsional electric field $T^{-1} \nabla T=-\mathbf{E}_{0}[42-44]$. As a direct consequence of this prescription, the anomalous thermal Hall current is given by $\mathbf{J}_{\epsilon}=T \mathbf{A}_{5} \times \nabla T / 6[6,40]$. In the equilibrium, the energy current vanishes [45] and Eq. (9) implies that

$$
\mathbf{A}_{5} \times \frac{\nabla T}{T}=\frac{1}{2} \nabla \times \mathbf{A}_{5} .
$$

This relation, coupling magnetization $\mathbf{A}_{5}=\left(k_{W} / M\right) \mathbf{M}(\mathbf{r})$ to thermal gradients, follows from Eq. (9) together with the expressions for magnetization-induced gauge and frame fields and is the main result of this Rapid Communication. It presents an alternative type of response with a topological origin and concrete observable effects.

To demonstrate the remarkable consequences of the TME, we consider a magnetic Weyl semimetal model, Eq. (1) with unidirectional magnetization $\mathbf{M}=\left[0,0, M_{3}(x)\right]$. In this case the Weyl node separation is parallel to $\mathbf{M}$ and Eq. (10) gives rise to the temperature profile

$$
\frac{T(x)}{T(0)}=\sqrt{\frac{K_{W}(0)}{K_{W}(x)},}
$$

where $K_{W}(x)=\sqrt{2 m\left(M_{3}(x)-m\right)}$. The relation Eq. (11) has a universal form and the material-specific details only enter through the dependence of Weyl node positions on magnetization. In this simple model, the magnetization-induced torsional electric field vanishes, but it is generally nonzero for time-dependent magnetization or interacting Weyl semimetals [46]. As shown in the SM, by replacing the node separation by its component perpendicular to the temperature gradient $k_{W} \rightarrow k_{W \perp}$, the relation (11) also applies to rotating Néel-type magnetic textures such as the one depicted in Fig. 2(a).

Experimental detection. Here, we discuss how our prediction linking the temperature profile to Weyl node separation, Eq. (11), can be detected in a topological insulator-magnetic insulator layer structure realization of Weyl semimetals $[15,16]$. In this case the magnetization perpendicular to the topological insulator layers is mapped to $M_{3}$ and our theory 


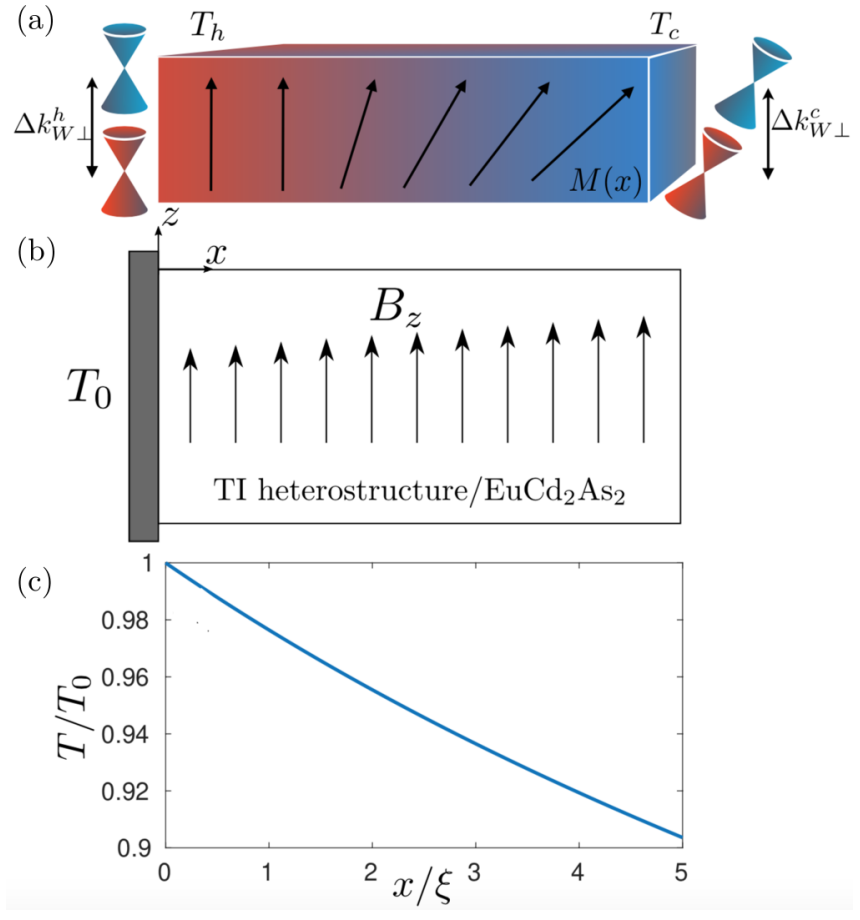

FIG. 2. (a) Néel-type textures in magnetic Weyl semimetals will induce temperature gradients. (b) A spatially varying magnetic field is applied in the $z$ direction, and the temperature at $x=0$ is fixed by a thermal bath. (c) Temperature as a function of position for the TI heterostructure setup. The parameter is $M_{3} / m=3+0.2 x / \xi$.

is readily applicable. The experimental setup is shown in Fig. 2(b). The inhomogeneous magnetization is realized by applying a decreasing magnetic field $B_{z}$ along the $x$ direction. There is no need for high-precision control over the field gradient as long as there is a detectable increase in magnetization over the sample. When the temperature at $x=0$ is fixed by a heat bath, there will be a temperature gradient along the $x$ direction. Figure 2(c) shows the result of the temperature as a function of position for $M_{3} / m=3+$ $0.2 x / \xi$. Alternatively, one could employ a naturally occurring Néel-type texture by contacting the sample to the heat bath near a domain wall and study the temperature profile in the vicinity.

Other candidate materials to observe this effect include ferromagnetic Weyl semimetals which have been recently identified experimentally [11-13,17-20] and are also captured by our theory. Specifically, we consider $\mathrm{EuCd}_{2} \mathrm{As}_{2}$, whose ground state is an itinerant magnet where $\mathrm{Eu}$ ions form ferromagnetic layers stacked antiferromagnetically along the $c$ axis. When a magnetic field is applied along the $c$ axis, $\mathrm{EuCd}_{2} \mathrm{As}_{2}$ becomes a Weyl semimetal with only two Weyl points $[11,12]$. Above the Néel temperature, the Weyl nodes have been observed [13] even in the absence of an external magnetic field, indicating strong ferromagnetic correlations. The bands near the Fermi level are dominated by the $\mathrm{Cd} 5 s$ and As $4 p$ orbitals. A theoretical description in terms of a $4 \times 4 \mathbf{k} \cdot \mathbf{p}$ Hamiltonian for the ferromagnetic state around the $\Gamma$ point has been constructed in Ref. [11]. The four- band model can be further mapped to Eq. (2) with $d_{1}=A k_{x}$, $d_{2}=A k_{y}$, and $d_{3}=M_{3}+m-C_{1} k_{z}^{2}$, where $m$ is the gap at $\Gamma$ point and $A$ and $C_{1}$ are material parameters (see the SM for details). The magnetization energy difference $M_{3}$ between the $p$ and $s$ orbitals arises from the exchange couplings between $\mathrm{Eu}$ magnetic moments and $\mathrm{Cd}$ and As orbitals. Thus, $M_{3}$ can be tuned by the spin polarization of $\mathrm{Eu}$ irons through an external magnetic field. Therefore, a position-dependent magnetic field can induce spatially varying $M_{3}$, which in turn leads to a temperature gradient through Eq. (11). Magnetization will directly couple to electrons so the temperature profile (11) refers to the electron temperature. When electronphonon mediated relaxation processes cannot be neglected, the predicted temperature profile will relax. However, at low temperatures the relaxation time becomes long and the temperature gradient can be observed after a bath temperature quench.

Conclusion and outlook. In summary, we developed an effective theory for magnetic Weyl semimetals where inhomogeneous magnetizations are mapped to spacetime torsion as well as axial gauge fields. Building on this notion, we discovered a different topological response, the thermal magnetorosional effect, which implies that an inhomogeneous magnetization will induce a temperature gradient in Weyl semimetals. This intriguing effect is rooted in the mixed chiral-torsional anomaly at finite temperature, which, unlike the zero temperature counterpart [2,3], is cutoff independent, and therefore is of a topological nature. We demonstrated how to detect this effect experimentally in recently discovered ferromagnetic Weyl semimetals as well as a TI-magnet heterostructure. Our discovery would be used as magnetic gradient refrigeration, and combining it with other thermoelectric effects provides other methods to control the responses of Weyl semimetals. In our prediction, the TME also provides an alternative and concrete way to experimentally verify the torsion anomaly [33], which has stirred controversy in the field theory literature [47-49].

In the present Rapid Communication we treated magnetization as a static field. Allowing for dynamical effects, our results imply that it is possible to manipulate magnetization by using temperature gradients. Furthermore, under those circumstances we expect exotic collective magnetic excitations resulting from the mixed chiral-torsional anomaly. To study these effects, the dynamics of the magnetization must be included in the effective theory, which we leave for future research. Another interesting issue for future study is to develop a theory of an elastic analog of the TME. Since our effective theory for Weyl fermions is analogous to the one obtained in the presence of lattice deformations [22], we expect that also strain would give rise to thermal effects. Torsion also emerges in topological superfluids and superconductors $[4,7,50]$, where the present theory may not be directly applicable because the central charge of the edge mode in that case [51] is different from the present model and the anomaly might be different. This presents another interesting avenue for future work.

Acknowledgments. The authors acknowledge the Aalto Center for Quantum Engineering for financial support. L.L. acknowledges useful discussions with Tao Jiang. 
[1] N. P. Armitage, E. J. Mele, and A. Vishwanath, Weyl and Dirac semimetals in three-dimensional solids, Rev. Mod. Phys. 90, 015001 (2018).

[2] O. Chandía and J. Zanelli, Topological invariants, instantons, and the chiral anomaly on spaces with torsion, Phys. Rev. D 55, 7580 (1997).

[3] O. Parrikar, T. L. Hughes, and R. G. Leigh, Torsion, parity-odd response, and anomalies in topological states, Phys. Rev. D 90, 105004 (2014).

[4] J. Nissinen and G. E. Volovik, On thermal Nieh-Yan anomaly in Weyl superfluid, arXiv:1909.08936.

[5] J. Nissinen and G. E. Volovik, On thermal Nieh-Yan anomaly in topological Weyl materials, JETP Lett. 110, 789 (2019).

[6] Z.-M. Huang, B. Han, and M. Stone, Nieh-Yan anomaly: Torsional Landau levels, central charge, and anomalous thermal Hall effect, Phys. Rev. B 101, 125201 (2020).

[7] J. Nissinen, Emergent Spacetime and Gravitational Nieh-Yan Anomaly in Chiral $p+i p$ Weyl Superfluids and Superconductors, Phys. Rev. Lett. 124, 117002 (2020).

[8] Z.-M. Huang, B. Han, and M. Stone, Hamiltonian approach to the torsional anomalies and its dimensional ladder, arXiv:1912.06051.

[9] A. Westström and T. Ojanen, Designer Curved-Space Geometry for Relativistic Fermions in Weyl Metamaterials, Phys. Rev. X 7, 041026 (2017).

[10] L. Liang and T. Ojanen, Curved spacetime theory of inhomogeneous Weyl materials, Phys. Rev. Research 1, 032006 (2019).

[11] L.-L. Wang, N. H. Jo, B. Kuthanazhi, Y. Wu, R. J. McQueeney, A. Kaminski, and P. C. Canfield, Single pair of Weyl fermions in the half-metallic semimetal EuCd ${ }_{2} \mathrm{As}_{2}$, Phys. Rev. B 99, 245147 (2019).

[12] J.-R. Soh, F. de Juan, M. G. Vergniory, N. B. M. Schröter, M. C. Rahn, D. Y. Yan, J. Jiang, M. Bristow, P. A. Reiss, J. N. Blandy, Y. F. Guo, Y. G. Shi, T. K. Kim, A. McCollam, S. H. Simon, Y. Chen, A. I. Coldea, and A. T. Boothroyd, Ideal Weyl semimetal induced by magnetic exchange, Phys. Rev. B 100, 201102(R) (2019).

[13] J.-Z. Ma, S. M. Nie, C. J. Yi, J. Jandke, T. Shang, M. Y. Yao, M. Naamneh, L. Q. Yan, Y. Sun, A. Chikina et al., Spin fluctuation induced Weyl semimetal state in the paramagnetic phase of $\mathrm{EuCd}_{2} \mathrm{As}_{2}$, Sci. Adv. 5, 4718 (2019).

[14] G. Y. Cho, Possible topological phases of bulk magnetically doped $\mathrm{Bi}_{2} \mathrm{Se}_{3}$ : Turning a topological band insulator into the Weyl semimetal, arXiv:1110.1939.

[15] A. A. Burkov and L. Balents, Weyl Semimetal in a Topological Insulator Multilayer, Phys. Rev. Lett. 107, 127205 (2011).

[16] A. A. Burkov, M. D. Hook, and L. Balents, Topological nodal semimetals, Phys. Rev. B 84, 235126 (2011).

[17] I. Belopolski, K. Manna, D. S. Sanchez, G. Chang, B. Ernst, J. Yin, S. S. Zhang, T. Cochran, N. Shumiya, H. Zheng, B. Singh, G. Bian, D. Multer, M. Litskevich, X. Zhou, S.-M. Huang, B. Wang, T.-R. Chang, S.-Y. Xu et al., Discovery of topological Weyl fermion lines and drumhead surface states in a room temperature magnet, Science 365, 1278 (2019).

[18] D. F. Liu, A. J. Liang, E. K. Liu, Q. N. Xu, Y. W. Li, C. Chen, D. Pei, W. J. Shi, S. K. Mo, P. Dudin, T. Kim, C. Cacho, G. Li, Y. Sun, L. X. Yang, Z. K. Liu, S. S. P. Parkin, C. Felser, and Y. L. Chen, Magnetic Weyl semimetal phase in a Kagomé crystal, Science 365, 1282 (2019).
[19] N. Morali, R. Batabyal, P. K. Nag, E. Liu, Q. Xu, Y. Sun, B. Yan, C. Felser, N. Avraham, and H. Beidenkopf, Fermiarc diversity on surface terminations of the magnetic Weyl semimetal $\mathrm{Co}_{3} \mathrm{Sn}_{2} \mathrm{~S}_{2}$, Science 365, 1286 (2019).

[20] H. Su, B. Gong, W. Shi, H. Yang, H. Wang, W. Xia, Z. Yu, P.-J. Guo, J. Wang, L. Ding, L. Xu, X. Li, X. Wang, Z. Zou, N. Yu, Z. Zhu, Y. Chen, Z. Liu, K. Liu, G. Li, and Y. Guo, Magnetic exchange induced Weyl state in a semimetal $\mathrm{EuCd}_{2}$ $\mathrm{Sb}_{2}$, arXiv:1903.12532.

[21] See Supplemental Material at http://link.aps.org/supplemental/ 10.1103/PhysRevResearch.2.022016 for technical details and derivations.

[22] A. Cortijo and M. A. Zubkov, Emergent gravity in the cubic tight-binding model of Weyl semimetal in the presence of elastic deformations, Ann. Phys. (NY) 366, 45 (2016).

[23] J. Yepez, Einstein's vierbein field theory of curved space, arXiv:1106.2037.

[24] K. Hayashi and T. Shirafuji, New general relativity, Phys. Rev. D 19, 3524 (1979).

[25] T. L. Hughes, R. G. Leigh, and E. Fradkin, Torsional Response and Dissipationless Viscosity in Topological Insulators, Phys. Rev. Lett. 107, 075502 (2011).

[26] Y. Hidaka, Y. Hirono, T. Kimura, and Y. Minami, Viscoelasticelectromagnetism and Hall viscosity, Prog. Theor. Exp. Phys. 2013, 013A02 (2013).

[27] T. L. Hughes, R. G. Leigh, and O. Parrikar, Torsional anomalies, Hall viscosity, and bulk-boundary correspondence in topological states, Phys. Rev. D 88, 025040 (2013).

[28] Y. You, G. Y. Cho, and T. L. Hughes, Response properties of axion insulators and Weyl semimetals driven by screw dislocations and dynamical axion strings, Phys. Rev. B 94, 085102 (2016).

[29] H. Sumiyoshi and S. Fujimoto, Torsional Chiral Magnetic Effect in a Weyl Semimetal with a Topological Defect, Phys. Rev. Lett. 116, 166601 (2016).

[30] M. N. Chernodub and M. A. Zubkov, Chiral anomaly in Dirac semimetals due to dislocations, Phys. Rev. B 95, 115410 (2017).

[31] Z. V. Khaidukov and M. A. Zubkov, Chiral torsional effect, JETP Lett. 108, 670 (2018)

[32] S. Imaki and A. Yamamoto, Lattice field theory with torsion, Phys. Rev. D 100, 054509 (2019).

[33] Y. Ferreiros, Y. Kedem, E. J. Bergholtz, and J. H. Bardarson, Mixed Axial-Torsional Anomaly in Weyl Semimetals, Phys. Rev. Lett. 122, 056601 (2019).

[34] Y. Ishihara, T. Mizushima, A. Tsuruta, and S. Fujimoto, Torsional chiral magnetic effect due to skyrmion textures in a Weyl superfluid ${ }^{3}$ He-A, Phys. Rev. B 99, 024513 (2019).

[35] Z.-M. Huang, L. Li, J. Zhou, and H.-H. Zhang, Torsional response and Liouville anomaly in Weyl semimetals with dislocations, Phys. Rev. B 99, 155152 (2019).

[36] H. T. Nieh and M. L. Yan, An identity in Riemann-Cartan geometry, J. Math. Phys. 23, 373 (1982).

[37] J. S. Bell and R. Jackiw, A PCAC puzzle: $\pi^{0} \rightarrow \gamma \gamma$ in the $\sigma$ model, Nuovo Cimento A 60, 47 (1969).

[38] S. L. Adler, Axial-vector vertex in spinor electrodynamics, Phys. Rev. 177, 2426 (1969).

[39] B. Bradlyn and N. Read, Topological central charge from Berry curvature: Gravitational anomalies in trial wave functions for topological phases, Phys. Rev. B 91, 165306 (2015). 
[40] K. Landsteiner, Notes on anomaly induced transport, Acta Phys. Pol., B 47, 2617 (2016).

[41] B. Bradlyn and N. Read, Low-energy effective theory in the bulk for transport in a topological phase, Phys. Rev. B 91, 125303 (2015).

[42] A. Shitade, Heat transport as torsional responses and Keldysh formalism in a curved spacetime, Prog. Theor. Exp. Phys. 2014, 123I01 (2014).

[43] G. Tatara, Thermal Vector Potential Theory of Transport Induced by a Temperature Gradient, Phys. Rev. Lett. 114, 196601 (2015).

[44] R. Nakai, S. Ryu, and K. Nomura, Laughlin's argument for the quantized thermal Hall effect, Phys. Rev. B 95, 165405 (2017).

[45] A. Kapustin and L. Spodyneiko, Absence of Energy Currents in an Equilibrium State and Chiral Anomalies, Phys. Rev. Lett. 123, 060601 (2019).
[46] J. Nissinen and G. E. Volovik, Type-III and IV interacting Weyl points, JETP Lett. 105, 447 (2017).

[47] E. W. Mielke and D. Kreimer, Chiral anomaly in contorted spacetimes, Gen. Relativ. Gravit. 31, 701 (1999).

[48] D. Kreimer and E. W. Mielke, Comment on "Topological invariants, instantons, and the chiral anomaly on spaces with torsion”, Phys. Rev. D 63, 048501 (2001).

[49] O. Chandía and J. Zanelli, Reply to "Comment on 'Topological invariants, instantons, and the chiral anomaly on spaces with torsion' ”, Phys. Rev. D 63, 048502 (2001).

[50] O. Golan and A. Stern, Probing topological superconductors with emergent gravity, Phys. Rev. B 98, 064503 (2018).

[51] N. Read and D. Green, Paired states of fermions in two dimensions with breaking of parity and time-reversal symmetries and the fractional quantum Hall effect, Phys. Rev. B 61, 10267 (2000). 